\title{
Provably Good Global Buffering by Generalized Multiterminal Multicommodity Flow Approximation
}

\author{
Feodor F. Dragan, Andrew B. Kahng, Ion I. Măndoiu, Sudhakar Muddu, and \\ Alexander Zelikovsky
}

\begin{abstract}
To implement high-performance global interconnect without impacting the placement and performance of existing blocks, the use of buffer blocks is becoming increasingly popular in structured-custom and block-based ASIC methodologies. Recent works by Cong, Kong, and Pan [5] and Tang and Wong [21] give algorithms to solve the buffer block planning problem. In this paper, we address the problem of how to perform buffering of global multiterminal nets given an existing buffer block plan. We give provably good and heuristic algorithms for this problem based on a recent approach of Garg and Könemann [9] and Fleischer [8] (see also Albrecht [1]). Our method routes connections using available buffer blocks, such that required upper and lower bounds on buffer intervals are satisfied. In addition, our algorithms allow more than one buffer to be inserted into any given connection and observe upper bounds and parity constraints on the number of buffers per connection. Most importantly, and unlike previous works on the problem [5], [21], we take into account (i) multiterminal nets, (ii) multiple routing layers, (iii) simultaneous buffered routing and compaction, and (iv) buffer libraries. Our method outperforms existing algorithms for the problem [5], which are based on 2-pin decompositions of the nets, and has been validated on top-level layouts extracted from a recent high-end microprocessor design.
\end{abstract}

This work was partially supported by Cadence Design Systems, Inc., the MARCO Gigascale Silicon Research Center and NSF Grant CCR-9988331.

F.F. Dragan is with the Department of Mathematics and Computer Science, Kent State University, Kent, OH 44242. E-mail: dragan@mcs.kent.edu.

A.B. Kahng is with the Departments of Computer Science and Engineering, and of Electrical and Computer Engineering, UC San Diego, La Jolla, CA 92093-0114. E-mail: abk@cs.ucsd.edu.

I.I. Măndoiu is with the Department of Computer Science, UC Los Angeles, Los Angeles, CA 90095-1596. E-mail: mandoiu@cc.gatech.edu.

S. Muddu is with Sanera Systems, Inc., Santa Clara, CA. E-mail: muddu@ sanera.net.

A. Zelikovsky is with the Department of Computer Science, Georgia State University, Atlanta, GA $30303 . \quad$ E-mail: alexz@cs.gsu.edu. 


\section{INTRODUCTION}

Process scaling leads to an increasingly dominant effect of interconnect on high-end chip performance. Each top-level global net must undergo repeater insertion (among other optimizations; see [4], [13], [16]) to maintain signal integrity and reasonable signal delay. ${ }^{1}$ Estimates of the need for repeater insertion range up to $10^{6}$ repeaters for top-level on-chip interconnect when we reach the $50 \mathrm{~nm}$ technology node. These repeaters are large (anywhere from $40 \times$ to $200 \times$ minimum inverter size), affect global routing congestion, can entail non-standard cell height and special power routing requirements, and can act as noise sources. In a block- or reuse-based methodology, designers seek to isolate repeaters for global interconnect from individual block implementations.

For these reasons, a buffer block methodology has become increasingly popular in structuredcustom and block-based ASIC methodologies. Two recent works by Cong, Kong, and Pan [5] and Tang and Wong [21] give algorithms to solve the buffer block planning problem. Their buffer block planning formulation is roughly stated as follows: Given a placement of circuit blocks, and a set of 2-pin connections with feasible regions for buffer insertion, ${ }^{2}$ plan the location of buffer blocks within the available free space so as to route a maximum number of connections.

In this paper, we address the problem of how to perform buffering of global nets given an existing buffer block plan. (Hence, our work is compatible with and complements the methods in [5], [21].) We give a provably good algorithm based on a recent approach of Garg and Könemann [9] and Fleischer [8]. Our method routes the nets using the available buffer blocks, such that required upper and lower bounds on repeater intervals - as well as length upper bounds per connectionare satisfied. ${ }^{3}$ Our algorithm allows more than one buffer to be inserted into any given connection and observes upper bounds on the number of buffers per connection. In addition, our algorithm observes repeater parity constraints, i.e., it will choose the number of inverters in any routing path according to the source and destination signal parity. The authors of [5], [21] assumed that global nets have been already decomposed into 2-pin connections; unlike these works our model takes into account multiterminal nets.

\footnotetext{
${ }^{1}$ Following the literature, we will use the terms buffer and repeater fairly interchangeably. When we need to be more precise: a repeater can be implemented as either an inverter or as a buffer (= two co-located inverters).

${ }^{2}$ In [21] only a single buffer per connection is allowed.

${ }^{3}$ For example, global repeater rules for a high-end microprocessor design in $0.25 \mu \mathrm{m}$ CMOS [12] require repeater intervals of at most $4500 \mu \mathrm{m}$. The number of buffers needed for a given connection depends strongly on the length of the connection; as noted in [12], the repeater interval is not only required for delay reduction, but also for crosstalk noise immunity and edge slewtime control.
} 
Our basic problem is informally defined as follows.

\section{Given:}

- a planar region with rectangular obstacles;

- a set of nets in the region, each net having:

- a single source and multiple sinks;

- a non-negative importance (criticality) coefficient;

- each sink having:

- a parity requirement, which specifies the required parity of the number of buffers (inverters) on the path connecting it to the source;

- a timing-driven requirement, which specifies the maximum number of buffers on the path to the source;

- a set of buffer blocks, each with given capacity; and

- an interval $[L, U]$ specifying lower and upper bounds on the distance between buffers.

Global Routing via Buffer Blocks (GRBB) Problem: route a subset of the given nets, with maximum total importance, such that:

- the distance between the source of a route and its first repeater, between any two consecutive repeaters, respectively between the last repeater on a route and the route's sink, are all between $L$ and $U$;

- the number of trees passing through any given buffer block does not exceed the block's capacity;

- the number of buffers on each source-to-sink path does not exceed the given upper bound and has the required parity; to meet the parity constraint two buffers of the same block can be used.

If possible, the optimum solution to the GRBB problem simultaneously routes all the nets. Otherwise, it maximizes the sum of the importance coefficients over routed nets. The importance coefficients can be used to model various practical objectives. For example, importance coefficients of 1 for each net correspond to maximizing the number of routed nets, and importance coefficients equal to the number of sinks of the net correspond to maximizing the number of connected sinks.

We also consider the following extensions of the basic GRBB problem: 
- Multi-Layer GRBB. The basic GRBB formulation imposes the same $L / U$ bounds on the length of all buffer-to-buffer, source-to-buffer, and buffer-to-sink wire segments. The multilayer GRBB problem accounts for the different electrical characteristics (unit-wire resistance and capacitance) of different routing layers and takes into consideration non-uniform source driving strengths and sink input capacitances.

- GRBB with Set Capacity Constraints. The basic GRBB problem assumes predetermined capacities for all buffer blocks. In practice, there is some freedom for transferring capacity from a buffer block to neighboring buffer blocks by translating circuit blocks. The GRBB problem with set capacity constraints captures this freedom by allowing constraints on the total capacity of sets of buffer blocks, instead of only constraining individual buffer blocks.

- GRBB with Buffer Library. To achieve better use of area and power resources, multiple buffer types can be used. The GRBB problem with buffer library optimally distributes the available buffer block capacity between given buffer types and simultaneously finds optimum buffered routings.

We give integer linear program (ILP) formulations for the basic GRBB problem and its extensions; these formulations generalize the vertex-capacitated integer multiterminal multicommodity flow (MTMCF) problem. The main contribution of the paper is a provably good algorithm for these generalizations of the MTMCF problem. Prior to our work, heuristics based on solving fractional relaxations of integer multicommodity flow formulations have been applied to VLSI global routing [15], [20], [2], [11], [1]. As noted in [14], the applicability of this approach is limited to problem instances of relatively small size by the prohibitive cost of solving exactly the fractional relaxation. As in the recent work of Albrecht [1], we avoid this limitation by using an approximation algorithm for solving the fractional relaxations. The approximation algorithm can find solutions within any desired accuracy; an important feature of the algorithm is that it allows for a smooth trade-off between runtime and solution accuracy. Our experiments indicate that even low accuracy fractional solutions give good final solutions for the GRBB problem after rounding.

The most interesting feature of our algorithm is its ability to work with multiterminal nets; previous work on the GRBB problem [5], [21] has considered only the case of 2-pin nets. Experiments on top-level layouts extracted from a recent high-end microprocessor design validate our algorithm, and indicate that it significantly outperforms existing algorithms for the problem based on 2-pin decompositions. 
The rest of the paper is organized as follows. In Section II we give ILP formulations for the GRBB problem and its extensions, and introduce a common generalization of these ILPs, referred to as the generalized multiterminal multicommodity flow (GMTMCF) ILP. The fractional relaxation of the GMTMCF ILP is a special type of packing LP, and can thus be approximated within any desired accuracy using the algorithm of Garg and Könemann [9]. In Section III we give a significantly faster approximation algorithm, obtained by extending a speed-up idea due to Fleischer [8] to this special type of packing LPs. We give the details of a key subroutine of the algorithmfinding minimum-weight feasible Steiner trees-in Section IV, and present algorithms for rounding near-optimal fractional GMTMCF solutions to near-optimal integral solutions in Section V. In Section VI we describe implementations of several GRBB heuristics, some based on rounding approximate fractional GMTMCF solutions, and some based on less sophisticated greedy ideas; Section VII gives the results of experiments comparing these heuristics on test cases extracted from the top-level layout of a recent high-end microprocessor. Finally, we conclude in Section VIII with a list of future research directions.

\section{INTEger LineAR PROgRAM Formulations}

Throughout this paper we let $N_{k}=\left(s_{k} ; t_{k}^{1}, \ldots, t_{k}^{q_{k}}\right), k=1, \ldots, K$, denote the nets to be routed; $s_{k}$ is the source, and $t_{k}^{1}, \ldots, t_{k}^{q_{k}}$ are the sinks of net $N_{k}$. We denote by $g_{k} \geq 1$ the importance (criticality) coefficient of net $N_{k}$, and by $a_{k}^{i} \in\{$ even, odd $\}$ and $l_{k}^{i} \geq 0$ the prescribed parity, respectively upper bound, on the number of buffers on the path between source $s_{k}$ and sink $t_{k}^{i}$. Let also $S=\left\{s_{1}, \ldots, s_{K}\right\}$ and $S^{\prime}=\left\{t_{1}^{1}, \ldots, t_{1}^{q_{1}}, \ldots, t_{K}^{1}, \ldots, t_{K}^{q_{K}}\right\}$ denote the set of sources, respectively of sinks, and $R=\left\{r_{1}, \ldots, r_{n}\right\}$ denote the given set of buffer blocks. For each buffer block $r_{i}$, we let $c\left(r_{i}\right)$ denote its capacity, i.e., the maximum number of buffers that can be inserted in $r_{i}$.

A routing graph for nets $N_{k}, k=1, \ldots, K$, is an undirected graph $G=(V, E)$ such that $S \cup S^{\prime} \subseteq V$. The set of vertices of $G$ other than sources and sinks, $V \backslash\left(S \cup S^{\prime}\right)$, is denoted by $V^{\prime}$. Specific routing graphs are defined in the following subsections for the GRBB problem and each of its extensions. All vertices in these routing graphs have associated locations on the chip, including those in $V^{\prime}$ which are associated with buffer block locations. The edges are defined according to the specific $L / U$ bounds imposed by each problem. Thus, every Steiner tree in $G$ automatically satisfies the given $L / U$ bounds assuming that a buffer is inserted at each Steiner point. To ensure that upperbound and parity constraints on the number of buffers on source-to-sink paths are met as well, we 
need to restrict the set of allowable Steiner trees as follows.

A path $p=\left(s_{k}, v_{1}, v_{2}, \ldots, v_{l}, t_{k}^{i}\right)$, connecting source $s_{k}$ to $\operatorname{sink} t_{k}^{i}$ in routing graph $G$, is a feasible $\left(s_{k}, t_{k}^{i}\right)$-path if

- $v_{i} \in V^{\prime}$ for each $i=1, \ldots, l$;

- the parity of $l$ is $a_{k}^{i}$; and

- $l \leq l_{k}^{i}$

A feasible Steiner tree for net $N_{k}$ is a Steiner tree $T_{k}$ in $G$ connecting terminals $s_{k}, t_{k}^{1}, \ldots, t_{k}^{q_{k}}$ such that, for every $i=1, \ldots, q_{k}$, the path of $T_{k}$ connecting $s_{k}$ to $t_{k}^{i}$ is a feasible $\left(s_{k}, t_{k}^{i}\right)$-path as defined above.

We will denote the set of all feasible Steiner trees for net $N_{k}$ by $\mathcal{T}_{k}$, and let $\mathcal{T}=\bigcup_{k=1}^{K} \mathcal{T}_{k}$. Given importance coefficients $g_{k}=g\left(N_{k}\right)$ for each net $N_{k}$, we define $g(T)=g_{k}$ for each tree $T \in \mathcal{T}_{k}$, $k=1, \ldots, K$.

\section{A. ILP Formulation of $G R B B$}

We begin by defining the routing graph $G=(V, E)$ for the GRBB problem. To allow feasible Steiner trees that meet parity constraints by using two buffers in the same buffer block, we introduce two distinct vertices, $r^{\prime}$ and $r^{\prime \prime}$, corresponding to each buffer block $r$, and define $V=S \cup S^{\prime} \cup\left\{r^{\prime}, r^{\prime \prime} \mid r \in R\right\}$. If $d(x, y)$ denotes the length of the shortest rectilinear path connecting points $x$ and $y$ and avoiding all given rectangular obstacles, the edge set of $G$ is defined by $E=E_{0} \cup E_{1}$, where

$$
\begin{aligned}
& E_{0}=\left\{\left(r^{\prime}, r^{\prime \prime}\right) \mid r \in R\right\} \\
& E_{1}=\{(x, y) \mid x, y \in V, L \leq d(x, y) \leq U\}
\end{aligned}
$$

The GRBB problem is then equivalent to the following integer linear program:

$\operatorname{maximize} \quad \sum_{T \in \mathcal{T}} g(T) f_{T}$

subject to

$$
\begin{aligned}
& \sum_{T \in \mathcal{T}} \pi_{T}(v) f_{T} \leq 1, \quad \forall v \in S \cup S^{\prime} \\
& \sum_{T \in \mathcal{T}}\left(\pi_{T}\left(r^{\prime}\right)+\pi_{T}\left(r^{\prime \prime}\right)\right) f_{T} \leq c(r), \quad \forall r \in R
\end{aligned}
$$




$$
f_{T} \in\{0,1\}, \quad \forall T \in \mathcal{T}
$$

where $\pi_{T}(v)$ is the number of occurrences of $v$ in $T$, i.e.,

$$
\pi_{T}(v)=\left\{\begin{array}{c}
0, \text { if } v \notin T \\
1, \text { if } v \in T
\end{array}\right.
$$

In (ILP1), the variable $f_{T}$ is set to 1 if the feasible Steiner tree $T$ is routed and to 0 otherwise. Constraints of the first type (corresponding to $v \in S \cup S^{\prime}$ ) ensure that at most one feasible Steiner tree is routed for each net; constraints of the second type (corresponding to $r \in R$ ) enforce buffer block capacities.

\section{B. ILP Formulation for Multi-Layer GRBB}

The basic version of the GRBB problem imposes identical $L / U$ bounds on the length of all buffer-to-buffer, source-to-buffer, and buffer-to-sink wire segments. This is not appropriate when routing is done in multiple layers, since different layers have different electrical characteristics (unit-wire resistance and capacitance). In addition, signal sources typically have non-uniform driving strengths, and signal sinks have non-uniform input capacitances. Thus, an accurate formulation of the GRBB problem for $z>1$ routing layers must handle:

- layer-dependent lower- and upper-bounds, $L_{i} / U_{i}, i=1, \ldots, z$, on the length of buffer-tobuffer wire-segments;

- source- and layer-dependent lower- and upper-bounds, $L_{i}^{s} / U_{i}^{s}, s \in S, i=1, \ldots, z$, on the length of source-to-buffer wire-segments; and

- sink- and layer-dependent lower- and upper-bounds, $L_{i}^{t} / U_{i}^{t}, t \in S^{\prime}, i=1, \ldots, z$, on the length of buffer-to-sink wire-segments.

These additional parameters are taken into account by appropriately modifying the routing graph graph $G=(V, E)$. The vertex set of $G$ remains the same, $V=S \cup S^{\prime} \cup\left\{r^{\prime}, r^{\prime \prime}: r \in R\right\}$, but we now define $E=E_{0} \cup E_{1} \cup E_{2} \cup E_{3}$, where

$$
\begin{aligned}
& E_{0}=\left\{\left(r^{\prime}, r^{\prime \prime}\right) \mid r \in R\right\} \\
& E_{1}=\left\{\left(s, r^{\prime}\right),\left(s, r^{\prime \prime}\right) \mid s \in S, r \in R, \exists i \in\{1, \ldots, z\} \text { s.t. } L_{i}^{s} \leq d_{i}(s, r) \leq U_{i}^{s}\right\} \\
& E_{2}=\left\{\left(r_{1}^{\prime}, r_{2}^{\prime}\right),\left(r_{1}^{\prime}, r_{2}^{\prime \prime}\right),\left(r_{1}^{\prime \prime}, r_{2}^{\prime}\right),\left(r_{1}^{\prime \prime}, r_{2}^{\prime \prime}\right) \mid r_{1}, r_{2} \in R, r_{1} \neq r_{2}, \exists i \in\{1, \ldots, z\} \text { s.t. } L_{i} \leq d_{i}\left(r_{1}, r_{2}\right) \leq U_{i}\right\} \\
& E_{3}=\left\{\left(r^{\prime}, t\right),\left(r^{\prime \prime}, t\right) \mid r \in R, t \in S^{\prime}, \exists i \in\{1, \ldots, z\} \text { s.t. } L_{i}^{t} \leq d_{i}(r, t) \leq U_{i}^{t}\right\}
\end{aligned}
$$


Here, $d_{i}(x, y)$ denotes the length of the shortest rectilinear path connecting points $x$ and $y$ and avoiding all rectangular obstacles in layer $i$.

The multi-layer GRBB problem is then equivalent to (ILP1) for the modified routing graph $G$.

\section{ILP Formulation for GRBB with Set Capacity Constraints}

Our basic formulation of the GRBB problem assumes predetermined capacities for all buffer blocks. In practice, buffer blocks are placed in the free space available after compaction, when some of the circuit blocks can still be moved within certain limits, thus transferring capacity from a buffer block to neighboring buffer blocks (see Fig. 1). This freedom is captured by upper-bounds on the total capacity of entire sets of buffer blocks, rather than individual buffer blocks.
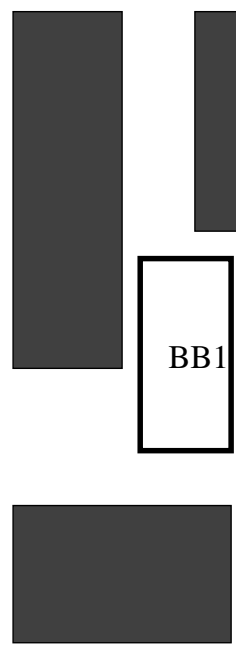
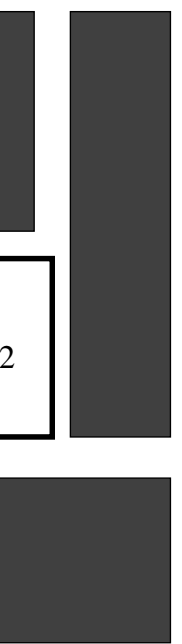

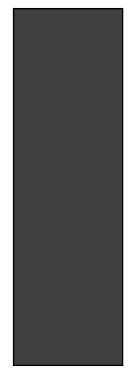

BB2
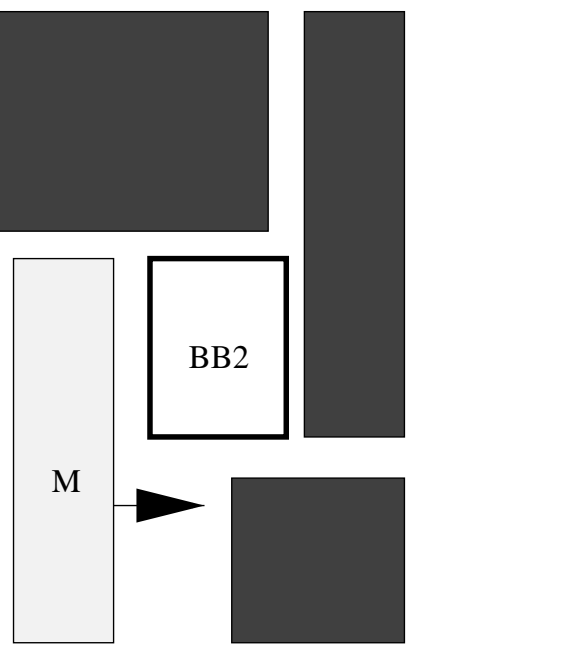
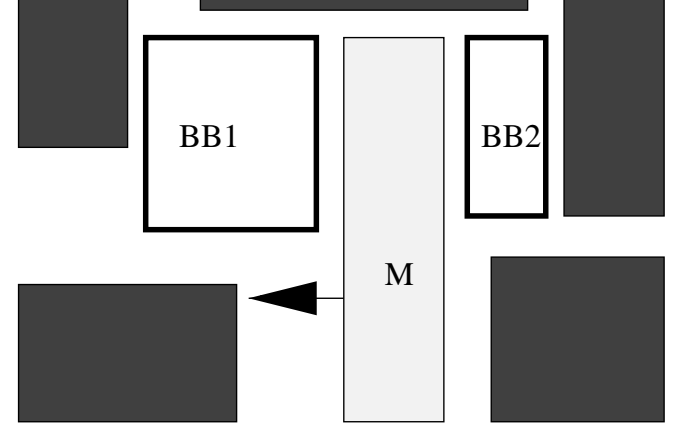

Fig. 1. Two buffer blocks BB1 and BB2 that share capacity: if the circuit block M moves right, then the capacity of buffer block BB1 is increasing while the capacity of buffer block BB2 is decreasing. In this example it is the sum of capacities of BB1 and BB2, rather than their individual capacities, that is bounded.

Assume that, as the result of compaction, we have identified subsets $R_{1}, \ldots, R_{p}$ of $R$ (some of which may consist of a single buffer block) such that there is a positive upper-bound $c\left(R_{i}\right)$ on the total capacity of each $R_{i}$. Assuming further that circuit block movements are so small compared to the given $L / U$ bounds that we can ignore changes in buffer block positions, it follows that the GRBB problem with set-capacity constraints given by $c\left(R_{1}\right), \ldots, c\left(R_{p}\right)$ is equivalent to the following integer linear program, in which the underlying routing graph is defined as in Section II-A. 
subject to

$$
\begin{aligned}
& \sum_{T \in \mathcal{T}} \pi_{T}(v) f_{T} \leq 1, \quad \forall v \in S \cup S^{\prime} \\
& \sum_{T \in \mathcal{T}}\left(\sum_{r \in R_{i}}\left(\pi_{T}\left(r^{\prime}\right)+\pi_{T}\left(r^{\prime \prime}\right)\right)\right) f_{T} \leq c\left(R_{i}\right), \quad \forall i \in\{1, \ldots, p\} \\
& f_{T} \in\{0,1\}, \quad \forall T \in \mathcal{T}
\end{aligned}
$$

\section{ILP Formulation for GRBB with Buffer Library}

The basic GRBB problem formulation implicitly assumes the use of a single buffer type. In practice, better use of area and power resources may be achieved by using more than one type of buffer. In this subsection we give an integer program formulation for the GRBB problem with buffers chosen from a given buffer library. This version of the problem allows buffered routings of the nets using any mix of buffers from the given library, and also allows buffers of different types (and hence, of different sizes) be placed in the same buffer block, up to the capacity of the block.

Let $B$ be the set of buffer types in the library. We assume to be given the size, size $(b)$, for each buffer type $b \in B$, as well as lower- and upper-bounds $L^{(s, b)} / U^{(s, b)}, L^{\left(b, b^{\prime}\right)} / U^{\left(b, b^{\prime}\right)}$, respectively $L^{(b, t)} / U^{(b, t)}$, on the length of each wire segment connecting source $s \in S$ to a buffer of type $b$, a buffer of type $b$ to a buffer of type $b^{\prime}$, respectively a buffer of type $b$ to $\operatorname{sink} t \in S^{\prime}$. To ensure that the available buffer block capacity is optimally distributed between the given buffer types and also allow feasible Steiner trees that use more than one buffer in the same buffer block, we introduce $2|B|$ vertices corresponding to each buffer block $r$. Formally, the routing graph $G=(V, E)$ has vertex set $V=S \cup S^{\prime} \cup\left\{r_{b}^{\prime}, r_{b}^{\prime \prime} \mid r \in R, b \in B\right\}$ and edge set $E=E_{0} \cup E_{1} \cup E_{2} \cup E_{3} \cup E_{4}$, where

$$
\begin{aligned}
& E_{0}=\left\{\left(r_{b}^{\prime}, r_{b}^{\prime \prime}\right) \mid r \in R, b \in B\right\} \\
& E_{1}=\left\{\left(r_{b_{1}}^{\prime}, r_{b_{2}}^{\prime}\right),\left(r_{b_{1}}^{\prime}, r_{b_{2}}^{\prime \prime}\right),\left(r_{b_{1}}^{\prime \prime}, r_{b_{2}}^{\prime}\right),\left(r_{b_{1}}^{\prime \prime}, r_{b_{2}}^{\prime \prime}\right) \mid r \in R, b_{1}, b_{2} \in B, b_{1} \neq b_{2}\right\} \\
& E_{2}=\left\{\left(s, r_{b}^{\prime}\right),\left(s, r_{b}^{\prime \prime}\right) \mid s \in S, r \in R, b \in B, L^{(s, b)} \leq d(s, r) \leq U^{(s, b)}\right\} \\
& E_{3}=\left\{\left(r_{b_{1}}^{\prime}, q_{b_{2}}^{\prime}\right),\left(r_{b_{1}}^{\prime}, q_{b_{2}}^{\prime \prime}\right),\left(r_{b_{1}}^{\prime \prime}, q_{b_{2}}^{\prime}\right),\left(r_{b_{1}}^{\prime \prime}, q_{b_{2}}^{\prime \prime}\right) \mid r, q \in R, r \neq q, b_{1}, b_{2} \in B, L^{\left(b_{1}, b_{2}\right)} \leq d(r, q) \leq U^{\left(b_{1}, b_{2}\right)}\right\} \\
& E_{4}=\left\{\left(r_{b}^{\prime}, t\right),\left(r_{b}^{\prime \prime}, t\right) \mid r \in R, b \in B, t \in S^{\prime}, L^{(b, t)} \leq d(r, t) \leq U^{(b, t)}\right\}
\end{aligned}
$$

The GRBB problem with buffer library $B$ is then equivalent to the following integer linear program: 
subject to

$$
\begin{aligned}
& \sum_{T \in \mathcal{T}} \pi_{T}(v) f_{T} \leq 1, \quad \forall v \in S \cup S^{\prime} \\
& \sum_{T \in \mathcal{T}}\left(\sum_{b \in B}\left(\pi_{T}\left(r_{b}^{\prime}\right)+\pi_{T}\left(r_{b}^{\prime \prime}\right)\right) \operatorname{size}(b)\right) f_{T} \leq c(r), \quad \forall r \in R \\
& f_{T} \in\{0,1\}, \quad \forall T \in \mathcal{T}
\end{aligned}
$$

\section{E. The Generalized MTMCF ILP}

Note that (ILP1-ILP3) are already strict generalization of previous integer edge-capacitated multiterminal multicommodity flow formulations used for VLSI global routing [19], [1], since they impose capacities on vertices and/or specific sets of vertices. In this subsection we formulate a common generalization of the integer linear programs (ILP1)-(ILP3), referred to as the generalized multiterminal multicommodity flow (GMTMCF) ILP, which allows (1) capacities on arbitrary sets of vertices, and (2) arbitrary vertex weights saying how much capacity is used by a tree visiting the vertex.

Given:

- nets $N_{k}, k=1, \ldots, K$, with importance coefficients $g_{k}$;

- a routing graph $G=(V, E)$ for the nets;

- arbitrary sets, $\mathcal{T}_{k}$, of Steiner trees for each net $N_{k}$;

- a family, $\mathcal{V}$, of subsets of $V$ such that $\{v\} \in \mathcal{V}$ for every $v \in S \cup S^{\prime}$;

- a function $s: V \rightarrow R_{+}$such that $s(v)=1$ for every $v \in S \cup S^{\prime}$; and

- a function $c: \mathcal{V} \rightarrow Z_{+}$such that $c(\{v\})=1$ for every $v \in S \cup S^{\prime}$;

the generalized multiterminal multicommodity flow ILP is:

maximize $\quad \sum_{T \in \mathcal{T}} g(T) f_{T}$

(GMTMCF ILP)

subject to

$$
\begin{array}{ll}
\sum_{T \in \mathcal{T}} \pi_{T}(X) f_{T} \leq c(X), & \forall X \in \mathcal{V} \\
f_{T} \in\{0,1\}, & \forall T \in \mathcal{T}
\end{array}
$$


where $\mathcal{T}=\cup_{k=1}^{K} \mathcal{T}_{k}, g(T)=g_{k}$ for every $T \in \mathcal{T}_{k}$, and

$$
\pi_{T}(X)=\sum_{v \in X} \pi_{T}(v) s(v)
$$

for every $T \in \mathcal{T}$ and $X \in \mathcal{V}$.

It is not difficult to see that (ILP1)-(ILP3) are special cases of the GMTMCF ILP. Thus, (ILP1) is obtained with $s \equiv 1$ by including in $\mathcal{V}$, besides singleton sets corresponding to sources and sinks, all sets $X_{r}=\left\{r^{\prime}, r^{\prime \prime}\right\}, r \in R$, and setting $c\left(X_{r}\right)=c(r)$. Similarly, (ILP2) is obtained with $s \equiv 1$ by including in $\mathcal{V}$ singleton sets corresponding to sources and sinks, as well as the sets $X_{i}=\left\{r^{\prime}, r^{\prime \prime} \mid r \in R_{i}\right\}, i=1, \ldots, p$, with $c\left(X_{i}\right)=c\left(R_{i}\right)$. Finally, (ILP3) is obtained for a family $\mathcal{V}$ containing singleton sets corresponding to sources and sinks together with the sets $X_{r}=\left\{r_{b}^{\prime}, r_{b}^{\prime \prime} \mid b \in\right.$ $B\}, r \in R$, for which $c\left(X_{r}\right)=c(r)$. In this case, $s(v)=1$ if $v$ is a source or a sink, and $s(v)=\operatorname{size}(b)$ if $v \in\left\{r_{b}^{\prime}, r_{b}^{\prime \prime} \mid r \in R\right\}$.

\section{ApPROXimAting THE GMTMCF LP RELAXATION}

Our two-step approach to the GRBB problem and its extensions is to (1) solve the fractional relaxations of (ILP1)-(ILP3), obtained by replacing integrality constraints $f_{T} \in\{0,1\}$ with $f_{T} \geq 0$, and then (2) use randomized rounding to get integer solutions. In this section we give an algorithm for approximating within any desired accuracy the fractional relaxation of the GMTMCF ILP, which subsumes (ILP1)-(ILP3). The algorithm relies on a subroutine for finding minimum weight feasible Steiner trees, the details of this subroutine are given in Section IV.

The fractional relaxation of the GMTMCF ILP, which we refer to as the generalized multiterminal multicommodity flow linear program (GMTMCF LP), can be solved exactly in polynomial time using, e.g., the ellipsoid algorithm. However, exact algorithms are highly impractical. On the other hand, the GMTMCF LP is a packing LP, and can thus be efficiently approximated within any desired accuracy using the recent combinatorial algorithm of Garg and Könemann [9]. We give a significantly faster approximation algorithm based on a speed-up idea originally proposed by Fleischer [8] for approximating the maximum edge-capacitated multicommodity flow (MCF).

Our algorithm simultaneously finds feasible solutions to the GMTMCF LP and its dual linear program. The dual LP asks for an assignment of non-negative weights $w(X)$ to every $X \in \mathcal{V}$ such that the weight of every tree $T \in \mathcal{T}$ is at least 1 , where the weight of $T$ is defined by weight $(T)=\frac{1}{g(T)} \sum_{X \in \mathcal{V}} \pi_{T}(X) w(X)$ : 
minimize $\sum_{X \in \mathcal{V}^{w}(X) c(X)}$

(GMTMCF Dual)

subject to

$$
\begin{array}{ll}
\frac{1}{g(T)} \sum_{X \in \mathcal{V}} \pi_{T}(X) w(X) \geq 1, & \forall T \in \mathcal{T} \\
w(X) \geq 0, & \forall X \in \mathcal{V}
\end{array}
$$

In the following we assume that $\min \left\{g_{k}: k=1, \ldots, K\right\}=1$ (this can be easily achieved by scaling) and denote $\max \left\{g_{k}: k=1, \ldots, K\right\}$ by $\Gamma$.

The algorithm (Figure 2) starts with weights $w(X)=\delta$ for every $X \in \mathcal{V}$, where $\delta$ is an appropriately chosen constant, and with a GMTMCF LP solution $f \equiv 0$. While there is a feasible tree whose weight is less than 1 , the algorithm selects such a tree $T$ and increments $f_{T}$ by 1 . This increase will likely violate the capacity constraints for some of the sets in $\mathcal{V}$; feasibility is achieved at the end of the algorithm by uniformly scaling down all $f_{T}$ 's. Whenever $f_{T}$ is incremented, the algorithm also updates each weight $w(X)$ by multiplying it with $\left(1+\varepsilon \pi_{T}(X) / c(X)\right)$, for a fixed $\varepsilon$.

According to the Garg and Könemann's approximation algorithm [9] each iteration must increment the variable $f_{T}$ corresponding to a tree with minimum weight among all trees in $\mathcal{T}$. Finding this tree essentially requires $K$ minimum-weight feasible Steiner tree computations, one for each net $N_{k}$. We reduce the total number of minimum-weight feasible Steiner tree computations during the algorithm by extending a speed-up idea due to Fleischer [8]. Instead of always finding the minimum-weight tree in $\mathcal{T}$, the idea is to settle for trees with weight within a factor of $(1+\varepsilon)$ of the minimum-weight. This speeds-up the computation since multiple variables $f_{T}$ can now be incremented, possibly more than once each, in a single iteration. As shown in next section, the faster algorithm still leads to an approximation guarantee similar to that of Garg and Könemann.

In each iteration the algorithm cycles through all nets. For each net, the algorithm repeatedly computes minimum-weight feasible Steiner tree until the weight becomes larger than $(1+\varepsilon)$ times a lower-bound $\bar{\alpha}$ on the overall minimum weight, $\min \{w e i g h t(T): T \in \mathcal{T}\}$. The lower-bound $\bar{\alpha}$ is initially set to $\delta / \Gamma$, and then multiplied by a factor of $(1+\varepsilon)$ from one iteration to another (note that no tree in $\mathcal{T}$ has weight smaller than $(1+\varepsilon) \bar{\alpha}$ at the end of an iteration, so $(1+\varepsilon) \bar{\alpha}$ is a valid lower-bound for the next iteration).

The scheme used for updating $\bar{\alpha}$ fully determines the number of iterations in the outer loop of the algorithm. Note that the lower-bound $\bar{\alpha}$ is at most $(1+\varepsilon)$ in the last iteration (since it increases 
Input: Nets $N_{1}, \ldots, N_{K}$, coefficients $g_{1}, \ldots, g_{K}$, routing graph $G=(V, E)$, family $\mathcal{V}$ of subsets of $V$, weights $c(X), X \in \mathcal{V}$, and $s(v), v \in V$

Output: GMTMCF LP solution $f_{T}, T \in \mathcal{T}$

For every $T \in \mathcal{T}, f_{T} \leftarrow 0$

For every $X \in \mathcal{V}, w(X) \leftarrow \delta$

$\bar{\alpha} \leftarrow \delta / \Gamma$ $/ / \bar{\alpha}$ is at all times a lower-bound on $\min \{\operatorname{weight}(T): T \in \mathcal{T}\}$

For $i=1$ to $t=\left\lfloor\log _{1+\varepsilon} \frac{(1+\varepsilon) \Gamma}{\delta}\right\rfloor$ do

For $k=1$ to $K$ do

Find a minimum weight feasible Steiner tree $T$ in $\mathcal{T}_{k}$

While weight $(T)<\min \{1,(1+\varepsilon) \bar{\alpha}\}$ do

$f_{T} \leftarrow f_{T}+1$

For all $X \in \mathcal{V}, w(X) \leftarrow w(X)\left(1+\varepsilon \pi_{T}(X) / c(X)\right)$

Find a minimum weight feasible Steiner tree $T$ in $\mathcal{T}_{k}$

End while

End for on $k$

$\bar{\alpha} \leftarrow(1+\varepsilon) \bar{\alpha}$

End for on $i$

For every $T \in \mathcal{T}, f_{T} \leftarrow \frac{f_{T}}{\log _{1+\varepsilon} \frac{(1+\varepsilon) \Gamma}{\delta}}$

Output $f_{T}, T \in \mathcal{T}$

Fig. 2. The algorithm for finding approximate solutions to the GMTMCF LP.

by a factor of $(1+\varepsilon)$ each time, and in the iteration before the last there is at least one tree of weight less than 1). Thus, since $\bar{\alpha}=\delta / \Gamma$ in the first iteration, the number of increases of $\bar{\alpha}$ is no larger than $\log _{1+\varepsilon} \frac{(1+\varepsilon) \Gamma}{\delta}$, and the final value of $i$ is $\left\lfloor\log _{1+\varepsilon} \frac{(1+\varepsilon) \Gamma}{\delta}\right\rfloor$.

\section{A. Runtime and Performance Analysis}

The two main loops of the algorithm (on $i$ and on $k$ ) are both repeated a fixed number of times, $\left\lfloor\log _{1+\varepsilon} \frac{(1+\varepsilon) \Gamma}{\delta}\right\rfloor$ and $K$, respectively. However, this does not immediately determine the runtime of the algorithm because of the variable number of iterations in the inner while loop. The following lemma gives an upper-bound on the runtime.

Lemma 1: Overall, the algorithm in Figure 2 requires $O\left(K \log _{1+\varepsilon} \frac{(1+\varepsilon) \Gamma}{\delta}\right)$ minimum-weight feasible Steiner tree computations. 
Proof. First, note that the number of minimum-weight feasible Steiner tree computations that do not contribute to the final fractional solution is $K\left\lfloor\log _{1+\varepsilon} \frac{(1+\varepsilon) \Gamma}{\delta}\right\rfloor$. Indeed, in each iteration, and for each net $N_{k}$, there is exactly one minimum-weight feasible Steiner tree computation revealing that $\min _{T \in \mathcal{T}_{k}}$ weight $(T) \geq(1+\varepsilon) \bar{\alpha}$, all other computations trigger the incrementation of some $f_{T}$.

We claim that the number of minimum-weight Steiner trees that lead to variable incrementations is at most $K \log _{1+\varepsilon} \frac{(1+\varepsilon) \Gamma}{\delta}$. To see this, note that the weight of the set $\left\{s_{k}\right\} \in \mathcal{V}$ is updated whenever a variable $f_{T}, T \in \mathcal{T}_{k}$, is incremented. Moreover, $w\left(\left\{s_{k}\right\}\right)$ is last updated when incrementing $f_{T}$ for a tree $T \in \mathcal{T}_{k}$ of weight less than one. Thus, before the last update, $w\left(\left\{s_{k}\right\}\right) \leq \Gamma \cdot$ weight $(T)<\Gamma$. Since $\pi_{T}\left(\left\{s_{k}\right\}\right)=c\left(\left\{s_{k}\right\}\right)=1$, the weight of $\left\{s_{k}\right\}$ is multiplied by a factor of $1+\varepsilon$ in each update, including the last one. This implies that the final value of $w\left(\left\{s_{k}\right\}\right)$ is at most $(1+\varepsilon) \Gamma$. Recalling that $w\left(\left\{s_{k}\right\}\right)$ is initially set to $\delta$, this gives that the number of updates to $w\left(\left\{s_{k}\right\}\right)$ is at most $\log _{1+\varepsilon} \frac{(1+\varepsilon) \Gamma}{\delta}$. The lemma follows by summing this upper-bound over all nets.

Lemma 2: The algorithm in Figure 2 computes a feasible solution to the GMTMCF LP.

Proof. We need to show that the values $f_{T}$ returned by the algorithm satisfy the inequality

$$
\sum_{T \in \mathcal{T}} \pi_{T}(X) f_{T} \leq c(X) \cdot \log _{1+\varepsilon} \frac{(1+\varepsilon) \Gamma}{\delta}
$$

for every $X \in \mathcal{V}$. Consider an arbitrary set $X \in \mathcal{V}$. Every time the variable $f_{T}$ is incremented by one unit, the weight of $X$ is also increased by a factor of $\left(1+\frac{x_{i} \varepsilon}{c(X)}\right)$, where $x_{i}=\pi_{T}(X)$. Using that $1+y \varepsilon \geq(1+\varepsilon)^{y}$ for $0 \leq y \leq 1$, we get that every sequence of updates with $\sum_{i} x_{i}=c(X)$ increases the weight of $X$ by a factor of at least

$$
\prod_{i}\left(1+\frac{x_{i} \varepsilon}{c(X)}\right) \geq \prod_{i}(1+\varepsilon)^{\frac{x_{i}}{c(X)}}=(1+\varepsilon)^{\frac{\sum_{i} x_{i}}{c(X)}}=1+\varepsilon
$$

Let $M=\sum_{T \in \mathcal{T}} \pi_{T}(X) f_{T}$. Since the initial weight of $X$ is $\delta$, from the previous inequality we get that the final weight of $X$ is at least $\delta(1+\varepsilon)^{\frac{M}{c(X)}}$.

Now, the last update of $w(X)$ is done when incrementing $f_{T}$ for a tree $T \in \mathcal{I}_{k}$ of weight less than one. Thus, the weight of $X$ is at most $g(T) \cdot$ weight $(T)<\Gamma$ before last update, and at most $(1+\varepsilon) \Gamma$ after. Combining this upper-bound on the final weight of $X$ with the lower-bound above gives that $\delta(1+\varepsilon)^{\frac{M}{c(X)}} \leq(1+\varepsilon) \Gamma$, i.e.,

$$
M \leq c(X) \log _{1+\varepsilon} \frac{(1+\varepsilon) \Gamma}{\delta}
$$


Finally, we show that, for an appropriate value of the parameter $\delta$, the feasible solution found by the algorithm is close to optimum.

Theorem 1: For every $\varepsilon<0.15$, the algorithm in Figure 2 computes a feasible solution to the GMTMCF LP within a factor of $1 /(1+4 \varepsilon)$ of optimum by choosing $\delta=(1+\varepsilon) \Gamma((1+\varepsilon) L \Gamma)^{-\frac{1}{\varepsilon}}$; the runtime of the algorithm for this value of $\delta$ is $O\left(\frac{1}{\varepsilon^{2}} K(\log L+\log \Gamma) T_{\text {tree }}\right)$. Here, $L$ is the maximum number of vertices in a feasible tree, and $T_{\text {tree }}$ is the time required to compute the minimum weight feasible Steiner tree for a net.

Proof. Our proof is an adaptation of the proofs of Garg and Könemann [9] and Fleischer [8]. We show that the solution computed by the algorithm is within a factor of $1 /(1+4 \varepsilon)$ of the optimum objective value, $\beta$, of the dual LP. The claimed approximation guarantee follows, since, by LP duality theory, $\beta$ is an upper-bound on the optimum objective value of the GMTMCF LP.

Let $\alpha(w)$ be the weight of a minimum weight tree from $\mathcal{T}$ with respect to weight function $w: \mathcal{V} \rightarrow R_{+}$, and let $D(w)=\sum_{X \in \mathcal{V}^{w}}(X) c(X)$. A standard scaling argument shows that the dual LP is equivalent to finding a weight function $w$ such that $D(w) / \alpha(w)$ is minimum, and that $\beta=$ $\min _{w}\{D(w) / \alpha(w)\}$.

For every $X \in \mathcal{V}$, let $w_{i}(X)$ be the weight of set $X$ at the end of the ith iteration and $w_{0}(X)=\delta$ be the initial weight of set $X$. For brevity, we will denote $\alpha\left(w_{i}\right)$ and $D\left(w_{i}\right)$ by $\alpha(i)$ and $D(i)$, respectively. Furthermore, let $f_{T}^{i}$ be the value of $f_{T}$ at the end of $i$ th iteration, and $h_{i}=\sum_{T \in \mathcal{T}} g(T) f_{T}^{i}$ be the objective value of the GMTMCF LP at the end of this iteration.

When the algorithm increments $f_{T}$ by one unit, each weight $w(X)$ is increased by $\left(\varepsilon \pi_{T}(X) w(X) / c(X)\right.$. Thus, the incrementation of $f_{T}$ increases $D(w)$ by

$$
\varepsilon \sum_{X \in \mathcal{V}} \pi_{T}(X) w(X)=\varepsilon \text { weight }(T) g(T)
$$

If this update takes place in the ith iteration, then weight $(T) \leq(1+\varepsilon) \alpha(i-1)$. Adding this over all $f_{T}$ 's incremented in $i$ th iteration gives

$$
D(i)-D(i-1) \leq \varepsilon(1+\varepsilon) \alpha(i-1)\left(h_{i}-h_{i-1}\right)
$$


which implies that

$$
D(i)-D(0) \leq \varepsilon(1+\varepsilon) \sum_{j=1}^{i} \alpha(j-1)\left(h_{j}-h_{j-1}\right)
$$

Consider the weight function $w_{i}-w_{0}$, and notice that $D\left(w_{i}-w_{0}\right)=D(i)-D(0)$. Since the minimum weight tree w.r.t. weight function $w_{i}-w_{0}$ has a weight of at $\operatorname{most} \alpha\left(w_{i}-w_{0}\right)+L \delta$ w.r.t. $w_{i}$, $\alpha(i) \leq \alpha\left(w_{i}-w_{0}\right)+L \delta$. Hence, if $\alpha(i)-L \delta>0$, then

$$
\beta \leq \frac{D\left(w_{i}-w_{0}\right)}{\alpha\left(w_{i}-w_{0}\right)} \leq \frac{D(i)-D(0)}{\alpha(i)-L \delta} \leq \frac{\varepsilon(1+\varepsilon) \sum_{j=1}^{i} \alpha(j-1)\left(h_{j}-h_{j-1}\right)}{\alpha(i)-L \delta}
$$

Thus, in any case (when $\alpha(i)-L \delta \leq 0$ this follows trivially) we have

$$
\alpha(i) \leq L \delta+\frac{\varepsilon(1+\varepsilon)}{\beta} \sum_{j=1}^{i} \alpha(j-1)\left(h_{j}-h_{j-1}\right)
$$

Note that, for each fixed $i$, the right-hand side of last inequality is maximized by setting $\alpha(j)$ to its maximum possible value, say $\alpha^{\prime}(j)$, for every $0 \leq j<i$. Then, the maximum value of $\alpha(i)$ is

$$
\begin{aligned}
\alpha^{\prime}(i) & =L \delta+\frac{\varepsilon(1+\varepsilon)}{\beta} \sum_{j=1}^{i-1} \alpha^{\prime}(j-1)\left(h_{j}-h_{j-1}\right)+\frac{\varepsilon(1+\varepsilon)}{\beta} \alpha^{\prime}(i-1)\left(h_{i}-h_{i-1}\right) \\
& =\alpha^{\prime}(i-1)\left(1+\frac{\varepsilon(1+\varepsilon)}{\beta}\left(h_{i}-h_{i-1}\right)\right) \\
& \leq \alpha^{\prime}(i-1) e^{\frac{\varepsilon(1+\varepsilon)}{\beta}\left(h_{i}-h_{i-1}\right)}
\end{aligned}
$$

where the last inequality uses that $1+x \leq e^{x}$ for every $x \geq 0$. Using that $\alpha^{\prime}(0)=L \delta$, this gives

$$
\alpha(i) \leq L \delta e^{\frac{\varepsilon(1+\varepsilon)}{\beta} h_{i}}
$$

Let $t$ be the last iteration of the algorithm. Since $\alpha(t) \geq 1$,

$$
1 \leq L \delta e^{\frac{\varepsilon(1+\varepsilon)}{\beta} h_{t}}
$$

and thus

$$
\frac{\beta}{h_{t}} \leq \frac{\varepsilon(1+\varepsilon)}{\ln (L \delta)^{-1}}
$$

Let $\gamma=\frac{\beta}{h_{t}} \log _{1+\varepsilon} \frac{(1+\varepsilon) \Gamma}{\delta}$ be the ratio between the optimum dual objective value and the objective value of the GMTMCF LP solution produced by the algorithm. By substituting the previous bound on $\beta / h_{t}$ we obtain

$$
\gamma \leq \frac{\varepsilon(1+\varepsilon) \log _{1+\varepsilon} \frac{(1+\varepsilon) \Gamma}{\delta}}{\ln (L \delta)^{-1}}=\frac{\varepsilon(1+\varepsilon) \ln \frac{(1+\varepsilon) \Gamma}{\delta}}{\ln (1+\varepsilon) \ln (L \delta)^{-1}}
$$


For $\delta=(1+\varepsilon) \Gamma((1+\varepsilon) L \Gamma)^{-\frac{1}{\varepsilon}}$,

$$
\frac{\ln \frac{(1+\varepsilon) \Gamma}{\delta}}{\ln (L \delta)^{-1}}=\frac{\ln ((1+\varepsilon) L \Gamma)^{\frac{1}{\varepsilon}}}{\ln ((1+\varepsilon) L \Gamma)^{-1+\frac{1}{\varepsilon}}}=\frac{\left.\frac{1}{\varepsilon} \ln (1+\varepsilon) L \Gamma\right)}{\left.\frac{1-\varepsilon}{\varepsilon} \ln (1+\varepsilon) L \Gamma\right)}=\frac{1}{1-\varepsilon}
$$

and thus

$$
\gamma \leq \frac{\varepsilon(1+\varepsilon)}{(1-\varepsilon) \ln (1+\varepsilon)} \leq \frac{\varepsilon(1+\varepsilon)}{(1-\varepsilon)\left(\varepsilon-\varepsilon^{2} / 2\right)} \leq \frac{(1+\varepsilon)}{(1-\varepsilon)^{2}}
$$

Here we use the fact that $\ln (1+\varepsilon) \geq \varepsilon-\varepsilon^{2} / 2$ (by Taylor series expansion of $\ln (1+\varepsilon)$ around the origin). The proof of the approximation guarantee is completed by observing that $(1+\varepsilon) /(1-$ $\varepsilon)^{2} \leq(1+4 \varepsilon)$ for every $\varepsilon<0.15$. The runtime follows by substituting $\delta$ in the bound given by Lemma 1.

\section{Computing Minimum-Weight Feasible Steiner Trees}

The key subroutine of the approximation algorithm given in the previous section is to compute, for a fixed $k$ and given weights $w(X), X \in \mathcal{V}$, a feasible tree $T \in \mathcal{T}_{k}$ minimizing weight $(T)=$ $\frac{1}{g(T)} \sum_{X \in \mathcal{V}} \pi_{T}(X) w(X)$. Define a weight function $w^{\prime}$ on the vertices of the routing graph $G=(V, E)$ by setting $w^{\prime}(v)=\frac{1}{g(T)} \sum_{v \in X \in \mathcal{V}^{w}} w(X)$, and let $w^{\prime}(T)=\sum_{v \in V(T)} w^{\prime}(v)$ be the total vertex weight w.r.t. $w^{\prime}$ of $T$. Then weight $(T)=w^{\prime}(T)$, and the problem reduces to finding a tree $T \in \mathcal{I}_{k}$ with minimum total vertex weight w.r.t. $w^{\prime}$.

Recall that for the GRBB problem and its extensions, $\mathcal{T}_{k}$ contains all Steiner trees connecting the source $s_{k}$ with the sinks $t_{k}^{1}, \ldots, t_{k}^{q_{k}}$ such that the number of intermediate vertices on each tree path between $s_{k}$ and $t_{k}^{i}$ has the parity specified by $a_{k}^{i}$ and does not exceed $l_{k}^{i}$. In this case we can further reduce the problem of finding the tree $T \in \mathcal{T}_{k}$ minimizing $w^{\prime}(T)$ to the minimum-cost directed rooted Steiner tree (DRST) problem in a directed acyclic graph $D_{k}$, defined as follows. Let $L_{k}=\max \left\{l_{k}^{1}, \ldots, l_{k}^{q_{k}}\right\}$ and $V^{\prime}=V(G) \backslash\left(S \cup S^{\prime}\right)$. Then

$$
V\left(D_{k}\right)=\left\{s_{k}\right\} \cup\left\{v_{j} \mid v \in V^{\prime}, 1 \leq j \leq L_{k}\right\} \cup\left\{t_{k}^{1}, \ldots, t_{k}^{q_{k}}\right\}
$$

and $E\left(D_{k}\right)=E_{1} \cup E_{2} \cup E_{3}$, where

$$
\begin{aligned}
& E_{1}=\left\{\left(s_{k}, v_{1}\right) \mid v \in V^{\prime},\left(s_{k}, v\right) \in E(G)\right\} \\
& E_{2}=\left\{\left(u_{j}, v_{j+1}\right) \mid u, v \in V^{\prime}, 1 \leq j<L_{k},(u, v) \in E(G)\right\} \\
& E_{3}=\left\{\left(u_{j}, t_{k}^{h}\right) \mid u \in V^{\prime}, 1 \leq h \leq q_{k}, 1 \leq j \leq l_{k}^{h}, j \equiv a_{k}^{h}(\bmod 2),\left(v, t_{k}^{h}\right) \in E(G)\right\}
\end{aligned}
$$


For a given directed graph $H=(X, U)$ with costs on arcs, a specified root $r \in X$, and a set of terminals $Y \subset X$, the directed rooted Steiner tree problem asks to find the minimum cost arborescence rooted at $r$ and spanning all the vertices in $Y$ (in other words $r$ should have a directed path to every vertex in $Y$ ). It is easy to see that finding a feasible Steiner tree $T \in \mathcal{T}_{k}$ with minimum $w^{\prime}(T)$ reduces to finding a minimum cost DRST in $D_{k}$ after assigning to each arc entering vertex $v_{i}, v \in V^{\prime}$, $1 \leq j \leq L_{k}$, a cost of $w^{\prime}(v)$, and to each arc entering sink $t_{k}^{h}, 1 \leq h \leq q_{k}$, a cost of $w^{\prime}\left(t_{k}^{h}\right)$.

Unfortunately, the minimum-cost DRST problem is NP-hard, and the fact that $D_{k}$ is acyclic does not help since there is a simple reduction for this problem from arbitrary directed graphs to acyclic graphs. As far as we know, the best result for the DRST problem, due to Charikar et al. [3], gives $O\left(\log ^{2} q_{k}\right)$-approximate solutions in quasi-polynomial time $O\left(n^{3 \log q_{k}}\right)$. Note, on the other hand, that the minimum-cost DRST can be found in polynomial time for small nets (e.g., in time $O\left(n^{M-1}\right)$ for nets with at most $M$ sinks, for $\left.M=2,3,4\right)$. Theorem 1 immediately gives

Corollary 1: If the maximum net size is $M \leq 4$, the algorithm in Figure 2 finds, for every $\varepsilon<$ 0.15 , a feasible solution to the GMTMCF LP within a factor of $1 /(1+4 \varepsilon)$ of optimum in time $O\left(\frac{1}{\varepsilon^{2}} K n^{M-1}(\log n+\log \Gamma)\right)$.

Since most of the nets in real designs have small size, Corollary 1 justifies the following practical strategy for finding approximate solutions to the GMTMCF LP: decompose nets with more than 4 pins into nets with 2-4 pins, then apply the approximation algorithm in Figure 2. Another heuristic approach to speed-up the computation, used by Albrecht [1] for edge-capacitated MTMCF approximation, is to compute (exactly or approximately) a DRST once, then use in each of the following iterations minimum directed spanning trees (with respect to the updated edge lengths) in the directed acyclic graph induced by $\left\{s_{k}, t_{k}^{1}, \ldots, t_{k}^{q_{k}}, p_{1}, \ldots, p_{s}\right\}$ in the metric closure of $D_{k}$, where $p_{1}, \ldots, p_{s}$ are the Steiner points of the original DRST. To find a minimum spanning directed tree in directed acyclic graphs, one can use a very simple procedure: for each vertex choose a shortest incoming arc, then, after running this procedure, recursively delete all leaves that are not sinks of the net $N_{k}$.

We have implemented both heuristics that use approximate DRSTs instead of optimum DRSTs and heuristics based on 2-, 3-, respectively 4-pin decompositions; results of experiments comparing these approaches are reported in Section VII. 


\section{ROUNDING THE Fractional GMTMCF}

In the previous two sections we presented an algorithm for computing near-optimal solutions to the GMTMCF LP. In this section we give two algorithms based on the randomized rounding technique of Raghavan and Thomson [18] (see also [14]) for converting near-optimal fractional GMTMCF solutions to near-optimal integer GMTMCF solutions, i.e., to near optimal buffered routings.

The first algorithm is given in Figure 3. Since the algorithm routes net $N_{k}$ with probability $f_{k}=$ $\sum_{T \in \mathcal{T}_{k}} f_{T}$, it follows that, on the average, the total importance of the nets routed by the algorithm is $\sum_{k=1}^{K} g_{k} f_{k}=\sum_{T \in \mathcal{T}} g(T) f_{T}$. By Theorem 1 , this is within a factor of $1 /(1+4 \varepsilon)$ of the optimum GMTMCF LP solution, which in turn is an upper-bound on the optimum GMTMCF ILP solution.

Input: Fractional GMTMCF solution $f_{T}, T \in \mathcal{T}$

Output: Routed trees, $T_{k} \in \mathcal{T}_{k}$

For each $k=1, \ldots, K$, select net $N_{k}$ with probability $f_{k}=\sum_{T \in \mathcal{T}_{k}} f_{T}$

Route each selected net $N_{k}$ by picking a tree $T_{k}$ from $\mathcal{T}_{k}$, where tree $T \in \mathcal{T}_{k}$ is picked with probability $f_{T} / f_{k}$

Fig. 3. Randomized GMTMCF rounding algorithm.

A potential drawback of the first rounding algorithm is that it requires the explicit representation of trees $T \in \mathcal{T}$ with $f(T) \neq 0$. Although the approximate GMTMCF algorithm produces a polynomial number of trees with non-zero $f_{T}$, storing all such trees is infeasible for large problem instances. Our second rounding algorithm (Figure 4) takes as input the net and edge cumulated GMTMCF values, $f_{k}=\sum_{T \in \mathcal{T}_{k}} f_{T}$, respectively $f_{k}(e)=\sum_{T \in \mathcal{T}_{k}: e \in E(T)} f_{T}$, thus using $O(K|E|)$ space. Note that the GMTMCF algorithm in Figure 2 can be easily modified to compute these cumulated GMTMCF values instead of $f_{T}$ 's.

Our second rounding algorithm routes net $N_{k}=\left(s_{k} ; t_{k}^{1}, \ldots, t_{k}^{q_{k}}\right)$ with the same probability as the first rounding algorithm and thus, as argued above, the total importance of the routed nets is within a factor of $1 /(1+4 \varepsilon)$ of optimum. The difference is in how each chosen net is routed: to route net $N_{k}$, the algorithm performs backward random walks from each sink of $N_{k}$ until reaching either the source $s_{k}$ or a vertex already connected to the source. The random walks are performed in the directed acyclic graphs $D_{k}$, with probabilities given by the normalized $f_{k}(e)$ values.

Ensuring that no set capacity is exceeded can be accomplished in two ways. One approach 
Input: Net- and edge-cumulated GMTMCF values, $f_{k}=\sum_{T \in \mathcal{T}_{k}} f_{T}$ and $f_{k}(e)=\sum_{T \in \mathcal{T}_{k}: e \in E(T)} f_{T}, k=1, \ldots, K$, $e \in E\left(D_{k}\right)$

Output: Routed trees $T_{k} \in \mathcal{T}_{k}$

For each $k=1, \ldots, K$, select net $N_{k}$ with probability $f_{k}$

Route each selected net $N_{k}$ as follows:

$T_{k} \leftarrow\left\{s_{k}\right\}$

For each sink $t_{k}^{i}$ in $N_{k}$ do

$P \leftarrow \emptyset ; \quad v \leftarrow t_{k}^{i}$

While $v \notin T_{k}$ do

Pick arc $(u, v)$ with probability $\frac{f_{k}(u, v)}{\sum_{(w, v) \in E} f_{k}(w, v)}$

$P \leftarrow P \cup\{(u, v)\} ; \quad v \leftarrow u$

End while

$T_{k} \leftarrow T_{k} \cup P$

End for

Fig. 4. Random walk based GMTMCF rounding algorithm.

is to solve the GMTMCF LP with set capacities scaled down by a small factor; this guarantees that the rounded solution meets the original capacities with very high probability (see [14] for an application of this approach to VLSI global routing). A simpler approach, extending the socalled greedy-deletion algorithm [6] to multiterminal nets, is to repeatedly drop routed nets passing through over-used sets until feasibility is achieved. We implement a modified version of the greedy-deletion algorithm in which, instead of dropping an entire tree, we drop only the sinks using paths through over-used sets.

\section{IMPLEMENTED AlgORITHMS}

In this section we describe the implemented algorithms for the GRBB problem.

\section{Greedy Routing Algorithms}

We have implemented four greedy algorithms for the GRBB problem, all of them based on the generic greedy routing algorithm given in Figure 5. All four greedy algorithms route nets sequentially. For a given net, the algorithms start with a tree containing only the net's source, then iteratively add shortest paths from each sink to the already constructed tree. The only difference is in whether or not net decomposition is used, and in the size of the decomposed nets. The first three algorithms - referred to as 2TG, 3TG, and 4TG, respectively — start by decomposing larger 
Input: Graph $G$ with $K$ nets $N_{1}, \ldots, N_{K}$, vertex capacities $c(v)$

Output: Fully or partially routed feasible Steiner trees $T_{k} \in \mathcal{T}_{k}$

For each $k=1, \ldots, K$, do

$T_{k} \leftarrow\left\{s_{k}\right\}$

For each sink $t_{k}^{i}$ in $N_{k}$ do

Using a backward BFS search, find a shortest path $P$ from $t_{k}^{i}$ to $T_{k}$ in $G$ using only vertices $v$ with $c(v)>0$; if no such path exists let $P=\emptyset$

$T_{k} \leftarrow T_{k} \cup P$

For each vertex $v$ in $P, c(v) \leftarrow c(v)-1$

End for

End for

Fig. 5. The generic greedy routing algorithm.

multiterminal nets into 2-, 3-, respectively 4-pin nets, and then apply the algorithm in Figure 5 to this decomposition. ${ }^{4}$ The fourth algorithm—which we refer to as MTG-is simply the algorithm in Figure 5 applied to the original (undecomposed) nets.

\section{GMTMCF Rounding Algorithms}

We have implemented four GMTMCF rounding algorithms, all of them based on the generic schema given in Figure 6. The first three algorithms-referred to as G2TMCF, G3TMCF, and G4TMCF, respectively_-start by decomposing larger multiterminal nets into 2-, 3-, respectively 4-pin nets, and then apply the generic GMTMCF routing algorithm to this decomposition. Since the optimum DRST can be efficiently computed for nets of these sizes, the three algorithms do not need to resort to the DRST approximations suggested at the end of Section IV. The fourth algorithm — henceforth referred to as GMTMCF_applies the flow rounding schema in Figure 6 to the undecomposed nets, using shortest-path trees as approximate DRSTs in the GMTMCF approximation step.

\section{IMPLEMENTATION EXPERIENCE}

All experiments were conducted on a SGI Origin 2000 with $16195 \mathrm{MHz}$ MIPS R10000 processors (only one of which is actually used by the sequential implementations included in our

\footnotetext{
${ }^{4}$ We remark that 2 TG is essentially the algorithm suggested in [5], except that in [5] shortest paths are computed in the forward direction, from sources toward sinks, and not from sinks toward sources as in Figure 5. It has been experimentally observed [7] that backward shortest paths give slightly better results than forward shortest paths.
} 
Input: Graph $G$ with $K$ nets $N_{1}, \ldots, N_{K}$, vertex capacities $c(v)$

Output: Fully or partially routed feasible Steiner trees $T_{k} \in \mathcal{T}_{k}$

Find an approximate GMTMCF using the algorithm in Figure 2

Round the approximate GMTMCF using the algorithm in Figure 4

Use greedy deletion to find a feasible integer solution

Use the MTG algorithm in Figure 5 on the unrouted nets to find a maximal routing

Fig. 6. The generic GMTMCF-based routing algorithm.

TABLE I

INSTANCE PARAMETERS.

\begin{tabular}{|c||cccccc|}
\hline ID & \#Nets & \#Sinks & Pins/net & L & U & BB Cap. \\
\hline h1 & 2396 & 2958 & 2.23 & 2000 & 4000 & 200 \\
h2 & 2438 & 3077 & 2.26 & 1000 & 4000 & 200 \\
h3 & 2448 & 3099 & 2.27 & 500 & 4000 & 200 \\
\hline i1 & 4764 & 6038 & 2.27 & 2000 & 4000 & 400 \\
i2 & 4925 & 6296 & 2.28 & 1000 & 4000 & 400 \\
i3 & 4938 & 6321 & 2.28 & 500 & 4000 & 400 \\
\hline
\end{tabular}

comparison) and 4 G-Bytes of internal memory, running under IRIX 6.4 IP27. Timing was performed using low-level Unix interval timers, under similar load conditions for all experiments. All algorithms were coded in $\mathrm{C}$ and compiled using gCC version egcs-2.91.66 with -04 optimization.

The six test cases used in our experiments were extracted from the next-generation (as of January 2000) microprocessor chip at SGI. We used an optimized floorplan of the circuit blocks and also optimized the location of the source/sink pin locations based on coarse timing budgets. We used $U=4000 \mu \mathrm{m}$, and varied $L$ between $500 \mu \mathrm{m}$ and $2000 \mu \mathrm{m}$. The upper-bounds on the number of buffers on paths from sources $s_{k}$ to sinks $t_{k}^{i}$ were computed with the formula $l_{k}^{i}=d\left(s_{k}, t_{k}^{i}\right) / 1000$. In all test cases considered the number of nets was large (up to 5000), and the number of buffer blocks small (50), with relatively large capacity (200-400 buffers per block); such values are typical for this application. Table I summarizes the parameters for the six test cases.

Tables II-V give the number of routed sinks and the runtime on the six test cases for each implemented algorithm. The results clearly demonstrate the high quality of solutions obtained by flow rounding methods. When applied to identical decompositions, flow-based methods yield improvements of 5-9\% in the number of connected sinks over the corresponding greedy algorithm. 


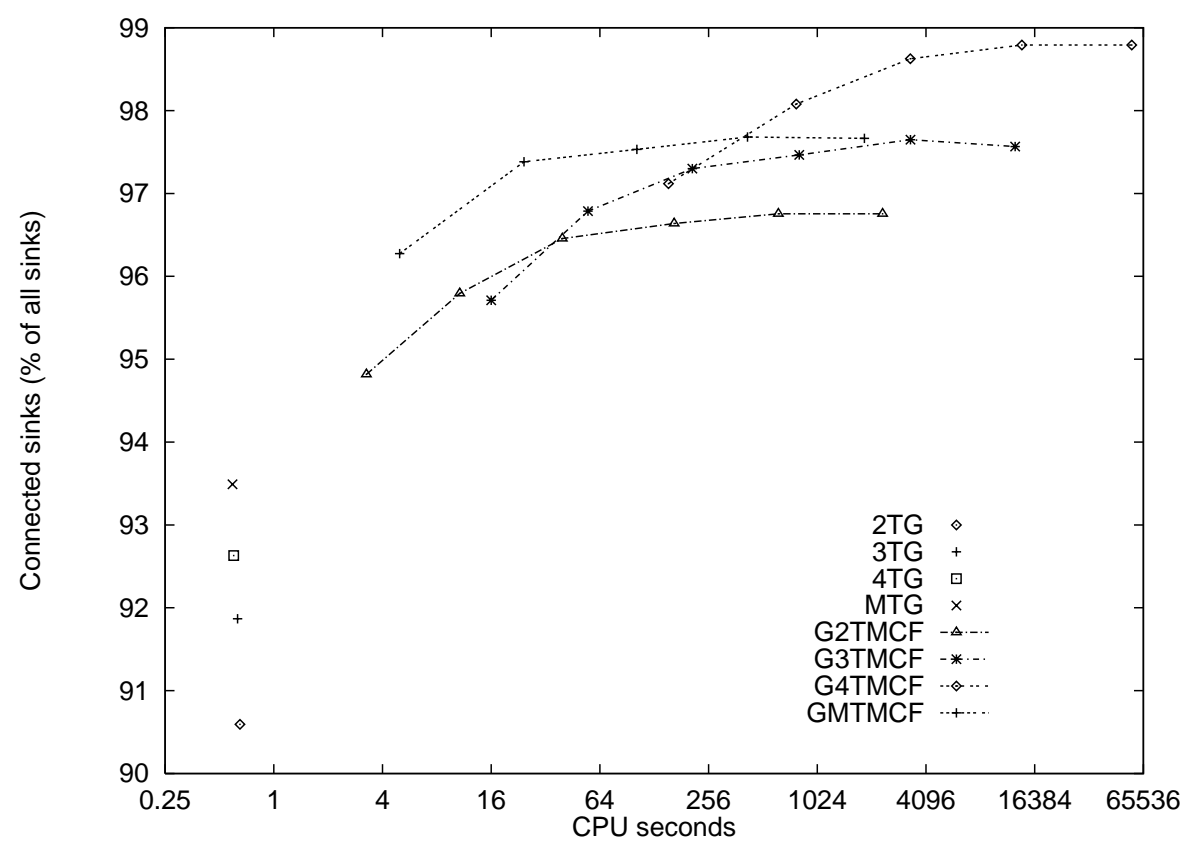

Fig. 7. Percent of sinks connected vs. CPU time on test case i1.

In fact, significant improvement over the best of the greedy methods is possible even with a very small increase in runtime, proof that even very coarse MCF/MTMCF approximations give helpful hints to the randomized rounding procedure.

Furthermore, the experimental results show that even a limited use of multiterminal nets (decomposition into nets of size 3 or 4 ) gives improvements over the already very high-quality solutions found by the flow-rounding algorithm based on 2-pin decompositions. More importantly, these improvements are observed even when the same time budget is given to the compared algorithms. To facilitate such a comparison, Figure 7 plots the solution quality versus the CPU time (in seconds, excluding I/O and memory allocation) of each algorithm when run on test case i1. The GMTMCF algorithm proves to be the best among all flow algorithms when the time budget is limited, providing significant improvements over greedy algorithms without undue runtime penalty. However, the best convergence to the optimum is achieved by G4TMCF, which dominates all other algorithms when high time budgets are allowed.

Table VI gives the amount of routing resources (buffers and wire area) used by each algorithm on test case i1. As expected, the amount of routing resources is higher for the algorithms with higher completion rates. In fact, even when normalizing by the number of connected sinks, the resource usage is slightly higher for these algorithms. This is at least partly explained by the fact 
TABLE II

PERCENT OF SINKS CONNECTED (BOLDFACE) AND CPU TIME FOR ALGORITHMS BASED ON 2-PIN DECOMPOSITION.

\begin{tabular}{|r||c|rrrrr|}
\hline \multirow{2}{*}{ ID } & \multirow{2}{*}{ 2TG } & \multicolumn{5}{|c|}{ G2TMCF } \\
\cline { 3 - 7 } & & $\varepsilon=0.64$ & $\varepsilon=0.32$ & $\varepsilon=0.16$ & $\varepsilon=0.08$ & $\varepsilon=0.04$ \\
\hline \hline \multirow{2}{*}{ h1 } & $\mathbf{8 8 . 3}$ & $\mathbf{9 3 . 7}$ & $\mathbf{9 5 . 4}$ & $\mathbf{9 5 . 4}$ & $\mathbf{9 5 . 7}$ & $\mathbf{9 5 . 5}$ \\
& 0.33 & 1.63 & 5.38 & 20.29 & 87.71 & 357.09 \\
\hline \multirow{2}{*}{ h2 } & $\mathbf{8 8 . 7}$ & $\mathbf{9 3 . 9}$ & $\mathbf{9 5 . 8}$ & $\mathbf{9 6 . 8}$ & $\mathbf{9 6 . 6}$ & $\mathbf{9 6 . 5}$ \\
& 0.37 & 2.35 & 5.99 & 22.78 & 88.44 & 349.63 \\
\hline \multirow{2}{*}{ h3 } & $\mathbf{8 8 . 4}$ & $\mathbf{9 3 . 5}$ & $\mathbf{9 5 . 4}$ & $\mathbf{9 6 . 5}$ & $\mathbf{9 6 . 4}$ & $\mathbf{9 5 . 5}$ \\
& 0.37 & 1.80 & 6.13 & 24.87 & 97.75 & 392.02 \\
\hline \multirow{2}{*}{ i1 } & $\mathbf{9 0 . 6}$ & $\mathbf{9 4 . 8}$ & $\mathbf{9 5 . 8}$ & $\mathbf{9 6 . 5}$ & $\mathbf{9 6 . 6}$ & $\mathbf{9 6 . 8}$ \\
& 0.65 & 3.26 & 10.71 & 39.61 & 164.85 & 622.87 \\
\hline \multirow{2}{*}{ i2 } & $\mathbf{9 1 . 6}$ & $\mathbf{9 6 . 2}$ & $\mathbf{9 7 . 1}$ & $\mathbf{9 7 . 4}$ & $\mathbf{9 7 . 5}$ & $\mathbf{9 7 . 6}$ \\
& 0.70 & 3.54 & 12.34 & 45.00 & 171.36 & 671.72 \\
\hline \multirow{2}{*}{ i3 } & $\mathbf{9 1 . 5}$ & $\mathbf{9 6 . 2}$ & $\mathbf{9 6 . 9}$ & $\mathbf{9 7 . 3}$ & $\mathbf{9 7 . 3}$ & $\mathbf{9 7 . 5}$ \\
& 0.73 & 3.57 & 11.84 & 47.17 & 172.01 & 770.51 \\
\hline
\end{tabular}

TABLE III

PERCENT OF SINKS CONNECTED (BOLDFACE) AND CPU TIME FOR ALGORITHMS BASED ON 3-PIN DECOMPOSITION.

\begin{tabular}{|r||c|rrrrr|}
\hline \multirow{2}{*}{ ID } & \multirow{2}{*}{3 TG } & \multicolumn{5}{|c|}{ G3TMCF } \\
\cline { 3 - 7 } & & $\varepsilon=0.64$ & $\varepsilon=0.32$ & $\varepsilon=0.16$ & $\varepsilon=0.08$ & $\varepsilon=0.04$ \\
\hline \hline \multirow{2}{*}{ h1 } & $\mathbf{9 0 . 2}$ & $\mathbf{9 6 . 2}$ & $\mathbf{9 7 . 1}$ & $\mathbf{9 7 . 3}$ & $\mathbf{9 7 . 7}$ & $\mathbf{9 7 . 8}$ \\
& 0.31 & 9.16 & 35.33 & 127.17 & 498.34 & 2090.61 \\
\hline \multirow{2}{*}{ h2 } & $\mathbf{9 0 . 1}$ & $\mathbf{9 6 . 4}$ & $\mathbf{9 8 . 3}$ & $\mathbf{9 8 . 6}$ & $\mathbf{9 8 . 9}$ & $\mathbf{9 8 . 5}$ \\
& 0.34 & 11.10 & 41.56 & 154.76 & 626.47 & 2355.66 \\
\hline \multirow{2}{*}{ h3 } & $\mathbf{8 9 . 8}$ & $\mathbf{9 6 . 4}$ & $\mathbf{9 7 . 7}$ & $\mathbf{9 8 . 3}$ & $\mathbf{9 8 . 1}$ & $\mathbf{9 8 . 0}$ \\
& 0.45 & 12.56 & 39.65 & 156.93 & 639.53 & 2364.51 \\
\hline \multirow{2}{*}{ i1 } & $\mathbf{9 1 . 9}$ & $\mathbf{9 5 . 7}$ & $\mathbf{9 6 . 8}$ & $\mathbf{9 7 . 3}$ & $\mathbf{9 7 . 5}$ & $\mathbf{9 7 . 6}$ \\
& 0.63 & 15.99 & 54.94 & 208.06 & 814.21 & 3362.20 \\
\hline \multirow{2}{*}{ i2 } & $\mathbf{9 2 . 7}$ & $\mathbf{9 7 . 0}$ & $\mathbf{9 8 . 0}$ & $\mathbf{9 8 . 4}$ & $\mathbf{9 8 . 5}$ & $\mathbf{9 8 . 6}$ \\
& 0.66 & 20.74 & 69.33 & 248.32 & 964.22 & 3834.26 \\
\hline \multirow{2}{*}{ i3 } & $\mathbf{9 2 . 5}$ & $\mathbf{9 6 . 8}$ & $\mathbf{9 7 . 8}$ & $\mathbf{9 8 . 3}$ & $\mathbf{9 8 . 4}$ & $\mathbf{9 8 . 4}$ \\
& 0.72 & 19.07 & 66.23 & 251.17 & 992.41 & 4164.50 \\
\hline
\end{tabular}


TABLE IV

PERCENT OF SINKS CONNECTED (BOLDFACE) AND CPU TIME FOR ALGORITHMS BASED ON 4-PIN DECOMPOSITION.

\begin{tabular}{|r||c|rrrrr|}
\hline \multirow{2}{*}{ ID } & \multirow{2}{*}{4 TG } & \multicolumn{5}{|c|}{ G4TMCF } \\
\cline { 3 - 7 } & & $\varepsilon=0.64$ & $\varepsilon=0.32$ & $\varepsilon=0.16$ & $\varepsilon=0.08$ & $\varepsilon=0.04$ \\
\hline \hline \multirow{2}{*}{ h1 } & $\mathbf{9 0 . 8}$ & $\mathbf{9 7 . 9}$ & $\mathbf{9 8 . 3}$ & $\mathbf{9 8 . 9}$ & $\mathbf{9 8 . 9}$ & $\mathbf{9 9 . 2}$ \\
& 0.31 & 56.16 & 305.70 & 1187.99 & 4881.40 & 19083.50 \\
\hline \multirow{2}{*}{ h2 } & $\mathbf{9 0 . 5}$ & $\mathbf{9 8 . 2}$ & $\mathbf{9 9 . 0}$ & $\mathbf{9 9 . 6}$ & $\mathbf{9 9 . 8}$ & $\mathbf{9 9 . 8}$ \\
& 0.34 & 75.98 & 364.02 & 1629.60 & 6520.44 & 24779.28 \\
\hline \multirow{2}{*}{ h3 } & $\mathbf{9 0 . 1}$ & $\mathbf{9 7 . 7}$ & $\mathbf{9 8 . 8}$ & $\mathbf{9 9 . 5}$ & $\mathbf{9 9 . 6}$ & $\mathbf{9 9 . 3}$ \\
& 0.33 & 75.26 & 392.30 & 1619.30 & 6378.52 & 25136.72 \\
\hline \multirow{2}{*}{ i1 } & $\mathbf{9 2 . 6}$ & $\mathbf{9 7 . 1}$ & $\mathbf{9 8 . 1}$ & $\mathbf{9 8 . 6}$ & $\mathbf{9 8 . 8}$ & $\mathbf{9 8 . 8}$ \\
& 0.60 & 153.46 & 782.65 & 3354.75 & 13910.05 & 56530.93 \\
\hline \multirow{2}{*}{ i2 } & $\mathbf{9 3 . 0}$ & $\mathbf{9 8 . 3}$ & $\mathbf{9 8 . 7}$ & $\mathbf{9 9 . 4}$ & $\mathbf{9 9 . 6}$ & $\mathbf{9 9 . 4}$ \\
& 0.71 & 191.14 & 1038.84 & 4550.27 & 17888.36 & 71636.67 \\
\hline \multirow{2}{*}{ i3 } & $\mathbf{9 2 . 8}$ & $\mathbf{9 8 . 2}$ & $\mathbf{9 8 . 6}$ & $\mathbf{9 9 . 3}$ & $\mathbf{9 9 . 3}$ & $\mathbf{9 9 . 3}$ \\
& 0.69 & 195.40 & 1062.71 & 4507.83 & 18438.52 & 73712.45 \\
\hline
\end{tabular}

TABLE V

PERCENT OF SINKS CONNECTED (BOLDFACE) AND CPU TIME FOR ALGORITHMS OPERATING ON UNDECOMPOSED NETS.

\begin{tabular}{|r||c|rrrrr|}
\hline \multirow{2}{*}{ ID } & \multirow{2}{*}{ MTG } & \multicolumn{5}{|c|}{ GMTMCF } \\
\cline { 3 - 7 } & & $\varepsilon=0.64$ & $\varepsilon=0.32$ & $\varepsilon=0.16$ & $\varepsilon=0.08$ & $\varepsilon=0.04$ \\
\hline \hline \multirow{2}{*}{ h1 } & $\mathbf{9 2 . 2}$ & $\mathbf{9 6 . 7}$ & $\mathbf{9 7 . 4}$ & $\mathbf{9 7 . 5}$ & $\mathbf{9 7 . 6}$ & $\mathbf{9 7 . 4}$ \\
& 0.30 & 2.33 & 11.21 & 47.11 & 223.96 & 946.78 \\
\hline \multirow{2}{*}{ h2 } & $\mathbf{9 2 . 3}$ & $\mathbf{9 7 . 6}$ & $\mathbf{9 8 . 9}$ & $\mathbf{9 9 . 2}$ & $\mathbf{9 9 . 3}$ & $\mathbf{9 9 . 3}$ \\
& 0.33 & 2.87 & 13.84 & 53.31 & 226.13 & 868.25 \\
\hline \multirow{2}{*}{ h3 } & $\mathbf{9 2 . 1}$ & $\mathbf{9 7 . 3}$ & $\mathbf{9 8 . 2}$ & $\mathbf{9 8 . 5}$ & $\mathbf{9 8 . 8}$ & $\mathbf{9 8 . 7}$ \\
& 0.33 & 2.86 & 12.66 & 53.74 & 219.20 & 876.63 \\
\hline \multirow{2}{*}{ i1 } & $\mathbf{9 3 . 5}$ & $\mathbf{9 6 . 3}$ & $\mathbf{9 7 . 4}$ & $\mathbf{9 7 . 5}$ & $\mathbf{9 7 . 7}$ & $\mathbf{9 7 . 7}$ \\
& 0.59 & 4.98 & 24.33 & 102.57 & 420.24 & 1865.81 \\
\hline \multirow{2}{*}{ i2 } & $\mathbf{9 3 . 6}$ & $\mathbf{9 7 . 7}$ & $\mathbf{9 8 . 1}$ & $\mathbf{9 8 . 2}$ & $\mathbf{9 8 . 3}$ & $\mathbf{9 8 . 4}$ \\
& 0.64 & 5.38 & 26.39 & 111.32 & 452.82 & 1827.98 \\
\hline \multirow{2}{*}{ i3 } & $\mathbf{9 3 . 3}$ & $\mathbf{9 7 . 7}$ & $\mathbf{9 8 . 1}$ & $\mathbf{9 8 . 1}$ & $\mathbf{9 8 . 2}$ & $\mathbf{9 8 . 2}$ \\
& 0.70 & 5.43 & 26.54 & 121.55 & 454.84 & 1833.17 \\
\hline
\end{tabular}


TABLE VI

USAGE OF ROUTING RESOURCES ON TEST CASE I1 (6038 SINKS TOTAL). THE MTG $\infty$ COLUMN GIVES RESOURCE USAGE FOR THE MTG ALGORITHM WHEN RUN WITH INFINITY CAPACITY FOR EACH BUFFER BLOCK.

\begin{tabular}{|c|c|c|c|c|c|c|c|c|c|c|c|c|c|}
\hline & \multicolumn{5}{|c|}{ Greed } & \multicolumn{2}{|c|}{ G2TMCF } & \multicolumn{2}{|c|}{ G3TMCF } & \multicolumn{2}{|c|}{ G4TMCF } & \multicolumn{2}{|c|}{ GMTMCF } \\
\hline & $2 \mathrm{TG}$ & $3 \mathrm{TG}$ & $4 \mathrm{TG}$ & MTG & $\mathrm{MTG}_{\infty}$ & $\varepsilon=0.64$ & $\varepsilon=0.04$ & $\varepsilon=0.64$ & $\varepsilon=0.04$ & $\varepsilon=0.64$ & $\varepsilon=0.04$ & $\varepsilon=0.64$ & $\varepsilon=0.04$ \\
\hline \#Conn. Sinks & 5469 & 5547 & 5592 & 5645 & 6038 & 5725 & 5842 & 5779 & 5896 & 5864 & 5965 & 5813 & 5897 \\
\hline$\%$ Conn. Sinks & 90.6 & 91.9 & 92.6 & 93.5 & 100 & 94.8 & 96.8 & 95.7 & 97.6 & 97.1 & 98.8 & 96.3 & 97.7 \\
\hline WL (meters) & 42.26 & 41.99 & 42.21 & 42.22 & 47.89 & 45.18 & 47.80 & 44.48 & 47.66 & 44.48 & 47.90 & 45.33 & 47.51 \\
\hline $\mathrm{WL} / \operatorname{Sink}(\mu \mathrm{m})$ & 7727 & 7570 & 7548 & 7479 & 7931 & 7891 & 8182 & 7697 & 8083 & 7585 & 8031 & 7798 & 8057 \\
\hline \#Buffers & 9240 & 9053 & 9076 & 9037 & 10330 & 9860 & 10676 & 9591 & 10610 & 9546 & 10730 & 9860 & 10647 \\
\hline \#Buffers/Sink & 1.69 & 1.63 & 1.62 & 1.60 & 1.71 & 1.72 & 1.83 & 1.66 & 1.80 & 1.63 & 1.80 & 1.70 & 1.81 \\
\hline
\end{tabular}

that higher completion rates can only be achieved by routing a larger percentage of "difficult" nets, which may otherwise be ignored. Note for example the increase in wirelength and average number of buffers per routed sink for the MTG algorithm when the completion rate is boosted by increasing buffer block capacities (columns MTG and $\mathrm{MTG}_{\infty}$ in Table VI).

\section{CONCLUSIONS AND Future DiRECTIONS}

In this paper, we addressed the problem of how to perform buffering of global multiterminal nets given an existing buffer block plan. We gave a provably good algorithm based on a novel approach to GMTMCF approximation inspired by recent results due to Garg and Könemann [9] and Fleischer [8]. Our GMTMCF algorithm outperforms existing algorithms for the problem [5], and has been validated on top-level layouts extracted from a recent high-end microprocessor design.

Ongoing work is aimed at increasing the space of methodologies to which our new techniques apply. As presented here, our work is clearly targeted to very early global wireplanning activity. In other words, the application domain is pre-synthesis chip planning: prescribed repeater intervals are driven only by coarse estimates of Miller coupling factors, repeater sizing, and source impedance or sink capacitance. The presented formulation also does not address timing criticalities or budgets except via net weighting; this is fortunately fairly common for initial wireplanning that breaks the "chicken-egg" problem of budgeting between-block and within-block paths in presynthesis RTL planning with aggressive global wire optimization. ${ }^{5}$ We are presently extending

\footnotetext{
${ }^{5}$ In other words, maximal repeater insertion allows maximum timing budgets for within-block timing paths, and this permits
} 
our approach to achieve better handling of timing criticality and budgets by improved use of net ordering and weighting during rounding, and post-processing of the solution to eliminate unneeded repeaters.

Further, we seek practical algorithms for handling routing congestion, i.e., simultaneously enforcing buffer block and channel capacities. By inserting "virtual" nodes corresponding to channels, the problem becomes a generalized type of integer GMTMCF in a vertex capacitated graph. However, computing minimum-weight feasible Steiner trees in this graph now entails finding minimum-weight length-restricted paths between buffer blocks. Although the latter problem is NP-hard, it can be approximated arbitrary close [10], [17] and it is still possible to apply our GMTMCF schema.

Finally, we note that actual applications would likely iterate the GRBB solution with incremental modification of buffer block locations, pin placements, and both channel and buffer block capacities. Adapting the GMTMCF approach and its runtime/quality profile for use in an iterative environment is challenging, and is the subject of ongoing collaboration with industry.

\section{REFERENCES}

[1] C. Albrecht, "Provably good global routing by a new approximation algorithm for multicommodity flow", Proc. ISPD, 2000.

[2] R.C. Carden and C.-K. Cheng, "A global router using an efficient approximate multicommodity multiterminal flow algorithm", Proc. DAC, 1991, pp. 316-321.

[3] M. Charikar, C. Chekuri, T. Cheung, Z. Dai, A. Goel, and S. Cheung, "Approximation algorithms for directed Steiner problems", J. Algorithms, 33 (1999), pp. 73-91.

[4] J. Cong, L. He, C.-K. Koh and P.H. Madden, "Performance optimization of VLSI interconnect layout", Integration 21 (1996), pp. 1-94.

[5] J. Cong, T. Kong and D.Z. Pan, "Buffer block planning for interconnect-driven floorplanning", Proc. ICCAD, 1999, pp. 358-363.

[6] F.F. Dragan, A.B. Kahng, I.I. Măndoiu, S. Muddu and A. Zelikovsky, "Provably good global buffering using an available buffer block plan”, Proc. ICCAD, 2000, pp. 104-109.

[7] F.F. Dragan, A.B. Kahng, I.I. Măndoiu, S. Muddu and A. Zelikovsky, "Provably good global buffering by multiterminal multicommodity flow approximation”, Proc. ASP-DAC, 2001, pp. 120-125.

[8] L.K. Fleischer, "Approximating fractional multicommodity flow independent of the number of commodities", Proc. 40th Annual Symposium on Foundations of Computer Science, 1999, pp. 24-31.

blocks to go through synthesis, place and route with more aggressive area targets. A strategy of uniform buffering of as many global nets as possible also helps control signal integrity and delay uncertainty problems. 
[9] N. Garg and J. Könemann, "Faster and simpler algorithms for multicommodity flow and other fractional packing problems", Proc. 39th Annual Symposium on Foundations of Computer Science, 1998, pp. 300-309.

[10] R. Hassin, "Approximation schemes for the restricted shortest path problem”, Math. Oper. Res., 17 (1992), pp. $36-42$.

[11] J. Huang, X.-L. Hong, C.-K. Cheng and E.S. Kuh, “An efficient timing-driven global routing algorithm”, Proc. DAC, 1993, pp. 596-600.

[12] A.B. Kahng, S. Muddu, E. Sarto and R. Sharma, "Interconnect tuning strategies for high-performance ICs", Proc. DATE, 1998.

[13] J. Lillis, C.K. Cheng and T.T.Y. Lin, "Optimal wire sizing and buffer insertion for low power and a generalized delay model", Proc. ICCAD, 1995, pp. 138-143.

[14] R. Motwani, J. Naor, and P. Raghavan, "Randomized approximation algorithms in combinatorial optimization", In Approximation algorithms for NP-hard problems (Boston, MA, 1997), D. Hochbaum, Ed., PWS Publishing, pp. 144-191.

[15] A.P.-C. Ng, P. Raghavan, and C.D. Thomson, "Experimental results for a linear program global router". Computers and Artificial Intelligence, 6 (1987), pp. 229-242.

[16] T. Okamoto and J. Cong, "Buffered Steiner tree construction with wire sizing for interconnect layout optimization”, Proc. ICCAD, 1996, pp. 44-49.

[17] C.A. Phillips, "The network inhibition problem”, Proc. 25th Annual ACM Symposium on Theory of Computing, 1993, pp. 776-785.

[18] P. Raghavan and C.D. Thomson, "Randomized rounding”, Combinatorica, 7 (1987), pp. 365-374.

[19] P. Raghavan and C.D. Thomson, "Multiterminal Global Routing: A Deterministic Approximation Scheme", Algorithmica, 6 (1991), pp. 73-82.

[20] E. Shragowitz and S. Keel, "A global router based on a multicommodity flow model”, Integration, 5 (1987), pp. $3-16$.

[21] X. Tang and D.F. Wong, "Planning buffer locations by network flows", Proc. ISPD, 2000. 